\title{
The origin of the 90 degree magneto-optical Kerr rotation in $\mathrm{CeSb}$
}

\author{
U. Pustogowa, W. Hübner, and K. H. Bennemann \\ Institute for Theoretical Physics, Freie Universität Berlin, Arnimallee 14, D-14195 Berlin, Germany
}

(October 14, 2018)

\begin{abstract}
We calculate the linear magneto-optical Kerr rotation for CeSb in the near-infrared spectral range. Using an exact formula for large Kerr rotation angles and a simplified electronic structure of CeSb we find at $\hbar \omega=0.46 \mathrm{eV}$ a Kerr rotation of $90^{\circ}$ which then for decreasing $\omega$ jumps to $-90^{\circ}$ as recently observed. We identify the general origin of possible $180^{\circ}$ polarization rotations as resulting from mainly nonmagnetic optical properties, in particular from the ratio of the dominant interband resonance frequency to the plasma frequency. The dependence of the Kerr rotation on moments and magnetization is discussed.
\end{abstract}

The search for large Kerr rotations in magneto-optics has been a longstanding subject of both basic and application-oriented research. The prediction and observation of giant Kerr rotations [1] in the nonlinear magneto-optical Kerr effect (NOLIMOKE) on multilayer sandwiches and thin magnetic films have successfully demonstrated the enhanced sensitivity of nonlinear optics to magnetism, in particular on low-dimensional systems due to the reduction of symmetry. Although the detailed values of the enhanced Kerr rotation in NOLIMOKE depend on the electronic structure of the investigated system, the spin-orbit coupling strength, the magnetization direction in the sample and the polarization of the incoming light, the $90^{\circ}$ nonlinear Kerr rotation in the longitudinal configuration and steep angle of incidence, is essentially independent of the frequency and results from symmetry considerations [4].

Prior and also parallel to NOLIMOKE, the search for enhanced Kerr rotations has been pursued in the conventional linear magneto-optic Kerr effect (MOKE). Since the MOKE rotation of transition metals like $\mathrm{Fe}$ or $\mathrm{Ni}$ is typically in the range of only some $0.1^{\circ}$ for optical frequencies one had to resort to particular magnetic rareearth or uranium alloys with large spin-orbit coupling constants, large magnetic moments, and with particular optical resonances in only one spin type to find relatively large Kerr rotations at low temperatures $(\approx 1 \mathrm{~K})$ and at lower frequencies. Furthermore, the application of large external magnetic fields $(\approx 5 \mathrm{~T})$ was necessary. In this way, a record-high MOKE rotation of $14^{\circ}$ has been observed for CeSb in 1986 by Schoenes et al. [5]. Recently, however, the same group (Pittini et al. [6]) observed the largest possible rotation of $90^{\circ}$ and an abrupt jump of this rotation to $-90^{\circ}$ in $\mathrm{CeSb}$ by reducing the frequency from $0.55 \mathrm{eV}$ to $0.46 \mathrm{eV}$.

Up to now, two groups performed first principle calculations of Kerr spectra of CeSb [7,8] both based on $\mathrm{LDA}+\mathrm{U}$ electronic structure calculations. Both emphasize as most important for near-infrared optics the interband transitions in hybridized $\mathrm{Sb} p$ bands and get similar diagonal and off-diagonal conductivities. Yaresko et al. [7] used an exact expression for large Kerr rotation angles but did not reproduce the $\pm 90^{\circ}$ rotation jump.
Liechtenstein et al. [8] did not calculate the Kerr rotation.

Thus, our aim is to explain the $\pm 90^{\circ}$ Kerr rotation in terms of the susceptibility tensor $\chi_{i j}$ and, in particular, to discuss the influence of interband and intraband parts of the electrical susceptibility using typical electronic properties of CeSb. We argue that this large Kerr rotation occurs for a frequency, which is set by both the plasma frequency and special optical transitions allowed only for one spin type. Thus, the Kerr rotation is not directly related to the magnetic properties. Our explanation relies largely on an improved evaluation of the Kerr rotation for a model band structure which does not assume any small parameters in the off-diagonal components of the reflected electric field. We use the result of $\mathrm{LDA}+\mathrm{U}$ calculations, in order to understand the effect of the Ce $f$ electrons spin-polarizing and splitting up the $\mathrm{Sb} p$ bands via hybridization. Our simplified theory outlined in the following attempts to describe the physical origin of the large linear Kerr effect.

The relation of MOKE signals (Kerr rotation or intensity measurements) to magnetic properties of the material is of special interest, since MOKE is used for the determination of the direction and relative strength of the magnetization in saturated ferromagnetic materials. By analyzing the reflectivity, the magnetic dichroism, and the electrical-susceptibility-tensor elements, we show that this special $90^{\circ}$ Kerr rotation is based on the behavior of the diagonal 'nonmagnetic' susceptibility $\chi_{x x}(\omega)$ and thus is not related to the magnitude of the magnetization in the sample.

To determine large Kerr rotation angles we have to use general expressions for the Kerr rotation $\varphi$ and ellipticity in contrast to the usually taken linearized formulae which are valid if $\tan \varphi \approx \varphi$ holds. Following general ellipsometry arguments the complex polar Kerr angle $\kappa$ is defined as

$$
\kappa=\frac{E_{y}}{E_{x}}=\frac{\tan \varphi+i \tan \varepsilon}{1-i \tan \varphi \tan \varepsilon},
$$

where, $\varphi$ is the Kerr rotation angle and $\varepsilon$ is the ellipticity. $E_{y}$ and $E_{x}$ are components of the reflected electric field. Solving Eq. (1) we find for the Kerr angle 


$$
\varphi=\arctan \left(-\frac{1-|\kappa|^{2}}{2 \operatorname{Re}(\kappa)} \pm \sqrt{\frac{\left(1-|\kappa|^{2}\right)^{2}}{4[\operatorname{Re}(\kappa)]^{2}}+1}\right) .
$$

The analysis of Eq. (2) yields that $180^{\circ}$-changes in $\varphi$ may occur when $\operatorname{Re}(\kappa)$ is zero or infinity. The behavior of $\kappa$ results from the well known expression for the linear polar Kerr rotation

$$
\kappa=-\frac{\chi_{x y}^{(1)}(\omega)}{\chi_{x x}^{(1)}(\omega)} \frac{1}{\sqrt{1+\chi_{x x}^{(1)}(\omega)}} .
$$

Here, $\chi_{x x}^{(1)}$ and $\chi_{x y}^{(1)}$ denote the elements of the linear susceptibility tensor [9]. The complex value $\kappa$ describes the tangent of the angle, $\kappa=\tan \Phi_{K}$, with $\Phi_{K}=\varphi+i \varepsilon$. Note, for small Kerr rotations Eq. (2) reduces to $\varphi=$ $\operatorname{Re}(\kappa)$. Eq. (2) can be rewritten as

$$
\varphi=\frac{1}{2} \arctan \left(\frac{2 \operatorname{Re}(\kappa)}{1-|\kappa|^{2}}\right)+\varphi_{0}
$$

with $\varphi_{0}=0$ for $|\kappa|^{2} \leq 1, \varphi_{0}=90^{\circ}$ for $|\kappa|^{2}>1, \operatorname{Re}(\kappa) \geq$ 0 , and $\varphi_{0}=-90^{\circ}$ for $|\kappa|^{2}>1, \operatorname{Re}(\kappa)<0$. The ellipticity angle $\varepsilon$ is given by

$$
\varepsilon=\frac{1}{2} \arcsin \left(\frac{2 \operatorname{Im}(\kappa)}{1+|\kappa|^{2}}\right) .
$$

For details see Groot Koerkamp [10,11].

Thus, the behavior of $\varphi$ for $\operatorname{Re}(\kappa) \rightarrow 0$ depends on the value of $|\kappa|^{2}$. For $|\kappa|^{2} \leq 1$, the case of small Kerr rotations, together with $\operatorname{Re}(\kappa)$ also $\varphi$ goes to zero. In the other case, for $|\kappa|^{2}>1$, changing the sign of $\operatorname{Re}(\kappa)$ yields two different values of $\varphi$, including a jump from $90^{\circ}$ to $-90^{\circ}$, since the Kerr rotation is defined only modulo $\pi$.

So far, Kerr effect measurements realized only the first case $|\kappa|^{2} \leq 1$ and no $\pm 90^{\circ}$ rotation angle was observed. Thus, we define two conditions for the occurence of a $\pm 90^{\circ}$ rotation angle $\varphi$

$$
\operatorname{Re}(\kappa)=0 \quad \text { and } \quad|\operatorname{Im}(\kappa)|>1
$$

Note, this was not explicitely discussed previously [12]. Here, these conditions are formulated for the calculationrelated values $\kappa$ and $\chi$. By means of the reflected perpendicularly polarized electric fields $E_{y}$ and $E_{x}$, Eq. (6) reproduces a complex phase of $90^{\circ}(\operatorname{see} \operatorname{Re}(\kappa)=0)$ and $E_{y 0}>E_{x 0}$ leading to $|\kappa|>1$.

In the following we describe a model for the electronic structure of CeSb. Thus, we analyze Eqs. (4) and (5) for realistic values of $\chi_{x x}$ and $\chi_{x y}$ in contrast to the discussions in refs. 3, [4] based on symmetry considerations.

For describing now the infrared Kerr-spectrum of CeSb as measured by Pittini et al. [6] we use only one feature of the electronic structure of CeSb close to the Fermi level, namely fairly flat and nearly parallel bands above and below the Fermi level $\varepsilon_{F}$ in the $\Gamma-\mathrm{Z}$ direction [8]. The $p$ states of $\mathrm{Sb}$ are split by approximately $0.6 \mathrm{eV}$. Furthermore, via hybridization with the $f$-electron minority-spin subband of $\mathrm{CeSb}$ (lying $\sim 3 \mathrm{eV}$ above $\varepsilon_{F}$ ) the $\mathrm{Sb} p$ states have an induced spin polarization. Thus, there are favored transitions of one spin sort between the $p$ states of very high weight 13]. In this case, the interband parts of the diagonal and off-diagonal susceptibilities can be written as sums over transitions for one spin band only. Thus,

$$
\chi_{x x}=\chi_{x x, \text { intra }}+\sum_{l, l^{\prime}} L_{l, l^{\prime}}, \quad \chi_{x y}=-i \frac{\lambda_{s . o .}}{\hbar \omega} \sum_{l, l^{\prime}} L_{l, l^{\prime}},
$$

where $\chi_{x x \text {,intra }}$ denotes the intraband contribution important only in the diagonal elements of $\chi, L_{l, l^{\prime}}$ denotes the response from transitions between the bands $l$ and $l^{\prime}$ and $\lambda_{\text {s.o. }}$ is the spin-orbit coupling constant. Note, the factor $\lambda_{\text {s.o. }} / \hbar \omega$ results from including spin-orbit coupling to lowest order in the wave functions. However, this will not directly affect $\varphi$. The Kerr rotation is then calculated by using

$$
\kappa=i \frac{\lambda_{\text {s.o. }}}{\hbar \omega} \frac{1}{\left(1+\frac{\chi_{x x, \text { intra }}}{L}\right) \sqrt{1+\chi_{x x}^{(1)}(\omega)}} .
$$

For simplicity, we approximate the transitions between the $(\mathrm{Sb}) p$ bands by a single atomic (nondispersive) Lorentzian

$$
\sum_{l, l^{\prime}} L_{l, l^{\prime}}=L:=\frac{C}{E_{f}-E_{i}-\hbar \omega+i \hbar \alpha},
$$

with the band positions $E_{f}$ of the unoccupied final state and $E_{i}$ of the occupied initial state, the damping factor $\alpha$, and the coefficient $C=\left(e^{2} M^{2} / V\right)$. In metals the symmetry of the transition-matrix elements $M$ plays a less important role and we use for simplicity the value $C=1$. Note, optical transitions between minority-spin-electron states dominate. Furthermore, we describe intraband effects by a conventional Drude term

$$
\chi_{x x, \text { intra }}=-\frac{\omega_{p l}^{2}}{\omega(\omega+i \tau)},
$$

with the plasma frequency $\omega_{p l}$ and the damping $\tau$. Using these expressions one can then determine $\varphi$ with the help of Eq. (4).

For a further analysis and for comparison with experiment we also calculate the optical reflectivity $\mathrm{R}$ using

$$
R=\left|\frac{N-1}{N+1}\right|^{2},
$$

with the refraction index $N^{2}=\epsilon_{0}+i \epsilon_{1}=1+\chi_{x x}+i \chi_{x y}$. 
We now present results for the Kerr rotation, the reflectivity, and in particular the dependence of the large Kerr angle on the plasma frequency $\omega_{p l}$ and interband splitting $\Delta E=E_{f}-E_{i}$ referring to the dominant optical transition.

In Fig. 1 the frequency dependence of the linear polar Kerr angle $\varphi$ and the ellipticity $\varepsilon$ are shown. Here $\varphi$ and $\varepsilon$ are calculated using Eqs. (4), (5), and (8). Parameters are choosen such as to obtain the change of $\varphi$ from $-90^{\circ}$ to $+90^{\circ}$ at precisely $0.46 \mathrm{eV}$ as measured by Pittini et al. [6]. Thus, we use for the interband splitting $\Delta E=$ $0.67 \mathrm{eV}$ in agreement with the bandstructure result of Liechtenstein et al. [8] and for the damping $\hbar \alpha=0.1 \mathrm{eV}$ as usually taken for Kerr angle calculations. In the Drude

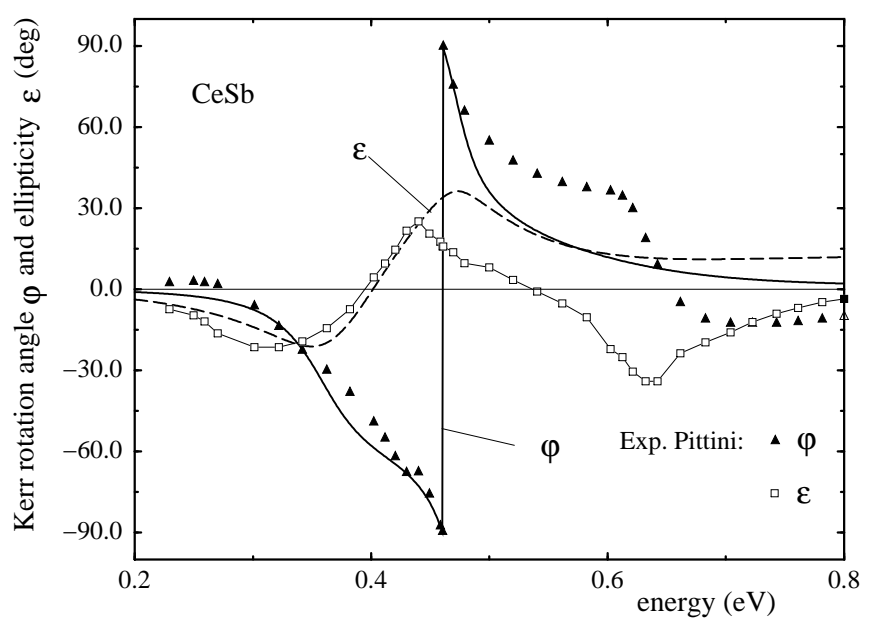

FIG. 1. Calculated frequency dependence of the linear magneto-optical Kerr-angle $\varphi$ (solid curve) and the ellipticity angle $\varepsilon$ (dashed curve) of CeSb calculated with a Lorentz-type susceptibility. For comparison also the experimental results of Pittini et al. [6] for $\varphi(\hbar \omega)$ and $\varepsilon(\hbar \omega)$ are shown.

term the parameters $\hbar \omega_{p l}=0.93 \mathrm{eV}$ and $\hbar \tau=0.95 \times$ $10^{-4} \mathrm{eV}$ are used. The plasma frequency is comparable with the calculated value of $1.1 \mathrm{eV}$ (see Ref. [7]). The value for $\tau$ is taken from Kwon et al. [14, but also values up to $10^{-1} \mathrm{eV}$ do not change the spectra. Note, the jump at $0.46 \mathrm{eV}$ is really a jump from an angle $-90^{\circ}$ to an angle $+90^{\circ}$ without intermediate values in contrast to the measurement with an external field of $3 \mathrm{~T}$ [6] (originated by the different magnetic phase of CeSb at $3 \mathrm{~T}$ and $5 \mathrm{~T}$ ) and in contrast to ab initio electronic structure based calculations [7]. The ellipticity angle changes sign at $0.41 \mathrm{eV}$ going from a minimum of $-20^{\circ}$ to the maximum of $35^{\circ}$. For comparison the experimental values by Pittini et al. [6] are shown as dots.

In Fig. 2 we show furthermore the frequency dependence of the optical reflectivity $R(\hbar \omega)$ in the energy range from 0 to $0.8 \mathrm{eV}$. For the frequency dependence of the Kerr angle the most important range of this curve is the deep minimum at $0.46 \mathrm{eV}$, at the same energy where the jump in $\varphi$ occurs. The discrepancy with respect to experimental results is presumably due to our simplified electronic structure of CeSb since we neglect interband transitions at other frequencies.

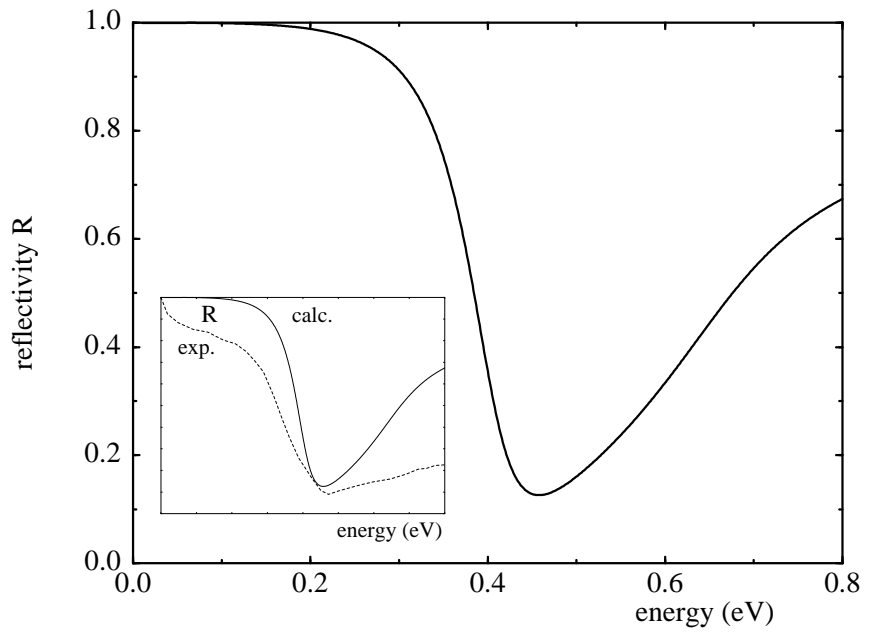

FIG. 2. Optical reflectivity $R$ of CeSb calculated from the diagonal susceptibility $\chi_{x x}(\omega)$. Experimental values are also shown.

Figs. 3 and 4 show the dependence of $\varphi(\hbar \omega)$ and, in particular, of the jump position and occurrence on $\Delta E$ and the plasma frequency $\omega_{p l}$. Fig. 3 shows Kerrangle spectra $\varphi(\hbar \omega)$ with plasma frequencies varying from $0.2 \mathrm{eV}$ to $1.3 \mathrm{eV}$. Note, for plasma frequencies higher than a threshold value depending on $\Delta E$ the $180^{\circ}$-jump vanishes, whereas for low plasma frequencies the jump amplitude is stable, while only the position of the jump moves to lower energies. The variation of the interband transition $\Delta E$ yields the opposite behavior, see Fig. 4. Here, $\Delta E$ changes from $0.2 \mathrm{eV}$ to $1.6 \mathrm{eV}$. The jump in $\varphi$ vanishes for low $\Delta E$ and moves for large values of $\Delta E$ to higher energies. Thus, we find that the ratio of the plasma frequency $\omega_{p l}$ to $\Delta E$, which characterizes the interband transitions, is essential for the occurrence of a $180^{\circ}$ jump in the Kerr angle.

The position of the $180^{\circ}$ abrupt change in the Kerr angle $\varphi$ is determined by a zero of the real part of $\chi_{x x}$, in particular $\left(\chi_{x x \text {,intra }}+L\right)=0$. This abrupt Kerr-angle change may occur. Note, this equation above reflects the ratio $\frac{\chi_{x x}}{\chi_{x y}}$. We used two approximations, namely (i) $\chi_{x y} \sim \frac{\lambda_{s . o}}{\hbar \omega}$ and (ii) assume interband transitions only in one spin subband. Within our band-structure model approximately for $\omega_{p l} / \Delta E \leq 1.5$ the abrupt Kerr angle change disappears. Moreover, there is no lower boundary for the occurrence of this change of the Kerr angle as a function of the ratio $\omega_{p l} / \Delta E[15]$.

For all parameter sets we analyzed the realization of the conditions in Eq.( 6 ). The first one, $\operatorname{Re}(\kappa)=0$ was 
fulfilled in every case (at different frequencies) indicating a resonance-like behavior in $\varphi$. The additional analysis

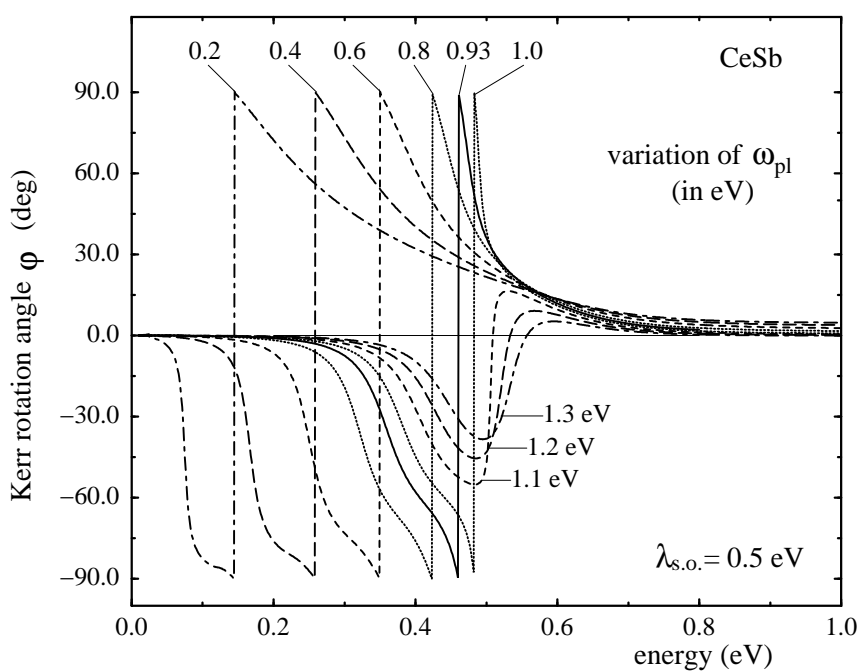

FIG. 3. Dependence of the Kerr angle spectra $\varphi(\hbar \omega)$ of $\mathrm{CeSb}$ on the value of the plasma frequency $\omega_{p l}$. For every curve the value of $\omega_{p l}$ (in $\mathrm{eV}$ ) is shown.

of the value of $|\kappa|$ or, since $\operatorname{Re}(\kappa)=0$, for $\operatorname{Im}(\kappa)$, enables us to distinguish between the already discussed resonances by Feil and Haas $\mid 16$ for $|\operatorname{Im}(\kappa)| \leq 1$ and the 'degenerate' resonances with the $\pm 90^{\circ}$ jump in $\varphi$ for $|\operatorname{Im}(\kappa)|>1$.

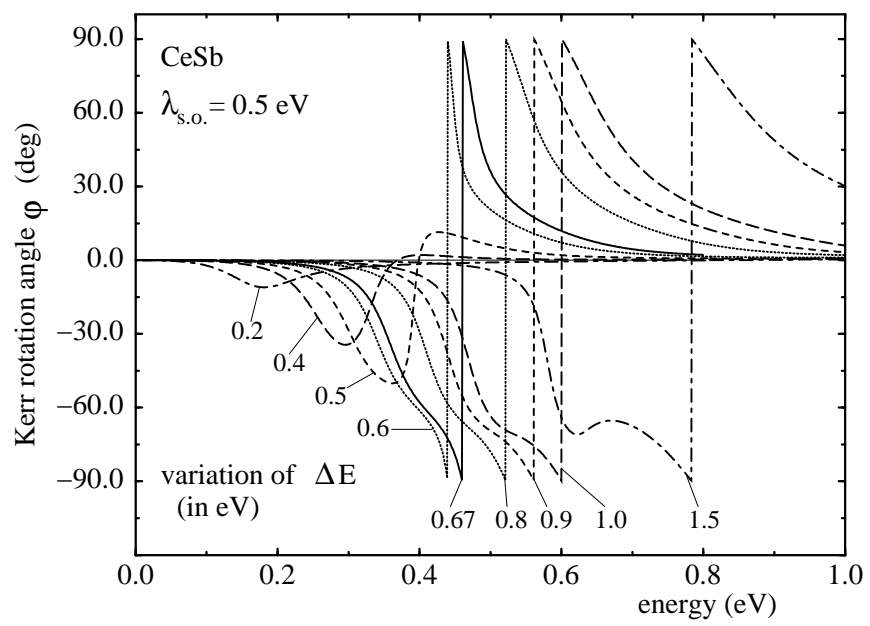

FIG. 4. Dependence of the Kerr angle spectra $\varphi(\hbar \omega)$ on the interband transition energy $\Delta E$. For every curve the corresponding value of $\Delta E$ (in $\mathrm{eV}$ ) is shown .

Note, the $\pm 90^{\circ}$ change in the Kerr rotation can be described also by a continuous rotation from $0^{\circ}$ to $180^{\circ}$, which are identical polarization angles. Then, the condition $|\operatorname{Im}(\kappa)|=1$ marks a distinct boundary between the two cases: (i) $|\operatorname{Im}(\kappa)| \leq 1$, then the polarization shows a resonance-like behavior at $\operatorname{Re}(\kappa)=0$ and returns back to the incident orientation direction and (ii) $|\operatorname{Im}(\kappa)|>1$, then a continuous rotation from $0^{\circ}$ to $180^{\circ}$ is allowed. The jump occurs only as a result of implementing the usual boundary conditions: $\varphi \rightarrow 0$ rotation angles outside the frequency region where $\operatorname{Re}(\kappa)=0$.

In the energy range below $\approx 0.5 \mathrm{eV}$ our results for the Kerr angle $\varphi(\hbar \omega)$ and Kerr ellipticity $\varepsilon(\hbar \omega)$ reproduce the experimental data very well. The deviation for larger energies results from the neglect of further interband transitions. Note, we have included only one interband transition with $\Delta E$ in our model. For the same reason, as already mentioned, our calculation yields a too large reflectivity at $0.8 \mathrm{eV}$. The inclusion of more interband transitions, especially of transitions of the other spin, would decrease the reflectivity in this energy range and yield results for the Kerr rotation, ellipticity and reflectivity in better agreement with experiment for larger energies.

Nevertheless, for the explanation of the $180^{\circ}$ Kerr angle jump it is not primarily important to know the exact electronic structure, but to fulfil the condition Eq.( 6). We do this by combining the important features of the electronic structure with the plasma frequency.

Historically, large linear Kerr rotations have been expected in materials with large spin-orbit coupling as in CeSb. In our model the spin-orbit coupling strength does of course influence the Kerr angle, since a large spin-orbit coupling is necessary to realize $\operatorname{Im}(\kappa)>1$, but does not influence the position determined by $\operatorname{Re}(\kappa)=0$ at which the Kerr rotation changes abruptly. In addition, in the case of CeSb the interband splitting $\Delta E$ is affected by the spin-orbit coupling. Note, for the Kerr effect in transition metals the spin-orbit interaction does not appreciably influence the electronic bandstructure, but of course has to be included in the wave functions.

Note, the origin of the $180^{\circ}$ Kerr rotation was discussed qualitatively in ref. 66. Dominant dipole transitions of only one spin type and low values of the optical constants were assumed. The first point we use directly in our model of the electronic structure of CeSb, whereas concerning the optical constants we formulate the exact conditions Eq. (6) and relate them quantitatively to $\chi_{i j}$ and the electronic structure. In contrast to ref. [6] we show the importance of the intraband part for the $180^{\circ}$ rotation, especially the quantitative relation of intra- to interband transitions at the 'jump' frequency required for the realization of the jump conditions, as illustrated in Figs. 3 and 4. However, note the formalism used in ref. 66 for the discussion of large Kerr rotation angles is based on the same electrodynamical expressions as in our derivation.

In view of our model, related materials with pronounced interband transitions due to flat $f$ and $d$ bands could exhibit similar behavior. However, due to the threshold character of the $\varphi$ enhancement and the Kerr- 
angle jump, slight changes of the parameters and dominant optical transitions might already suppress the giant Kerr effect.This may explain why for example CeBi with similar $\lambda_{\text {s.o. }}$ and magnetic moments exhibits only a Kerr angle of about $-9^{\circ}$ [18]. The different shapes of $\sigma_{x y}(\omega)$ for $\mathrm{CeSb}$ and $\mathrm{CeBi}$ might suggest already differences in the dominant dipole transitions. In addition, as discussed before, these dominant interband transitions in CeSb are explained by hybridized $p$ bands of Sb.

Most interesting is the relation of the discussed Kerrrotation jump to the magnetic properties of the material. In view of this we calculate the magnetic contrast $d$ for MOKE,

$$
d=\frac{I(+M)-I(-M)}{I(+M)+I(-M)}
$$

where $I( \pm M)$ are the intensities for reversed magnetization directions. Using for the intensities of the reflected light $I=\left|E_{\text {refl }}\right|^{2}=\left|\chi E_{\text {incident }}\right|^{2}$ we find $I( \pm M) \sim$ $\left|\chi_{x x} \pm i \chi_{x y}\right|^{2}$ and for the magnetic contrast

$$
d=2 \frac{\operatorname{Im}\left(\chi_{x x}\right) \operatorname{Re}\left(\chi_{x y}\right)-\operatorname{Re}\left(\chi_{x x}\right) \operatorname{Im}\left(\chi_{x y}\right)}{\left|\chi_{x x}\right|^{2}+\left|\chi_{x y}\right|^{2}} .
$$

In Fig. 5 we illustrate the different frequency dependences of the magnetic contrast $d$ and the absorptive part of the magnetic susceptibility tensor element $\operatorname{Re}\left(\chi_{x y}\right)$. We find a strong contrast of more than $70 \%$ near the frequency of the abrupt change in $\varphi$ where the spin-polarized absorption depends only on the electronic structure. The maximum in $\operatorname{Re}\left(\chi_{x y}\right)$ occurs at $\Delta E=0.67 \mathrm{eV}$. In previous more detailed microscopic calculations of $\chi_{x y}[19$ we found a linear dependence of amplitudes of pronounced maxima in $\chi_{x y}$ on the magnetic moment, which seems to be a general property. On

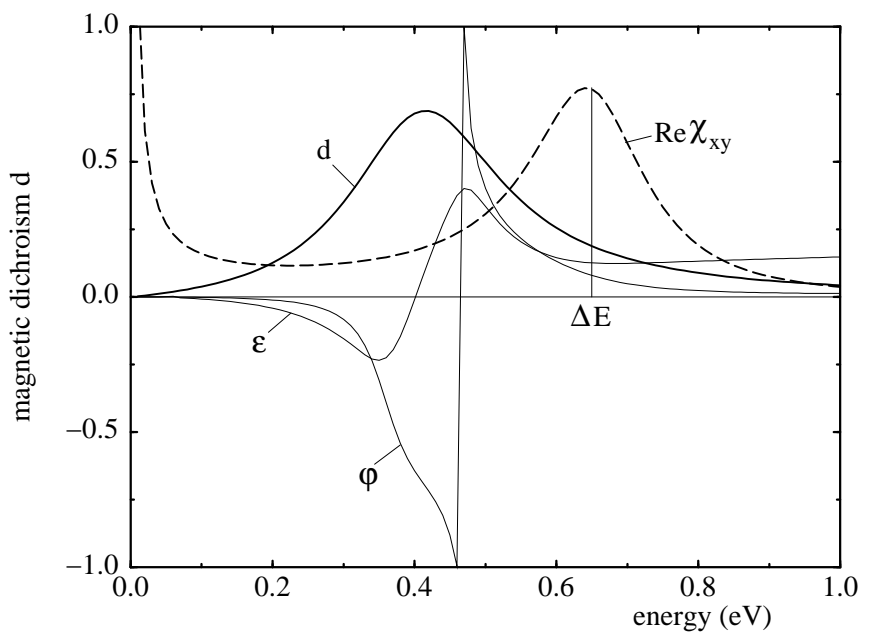

FIG. 5. Frequency dependence of the magnetic dichroism $d$ and the absorptive part of the magnetic susceptibility $\operatorname{Im}\left(\chi_{x y}\right)$. the other hand, inspecting Eq. (3) (small Kerr effect) for a weakly varying denominator we find $\varphi \sim \chi_{x y} \sim M$. For the $\pm 90^{\circ}$ change in $\varphi$, by contrast, the Kerr rotation as well as the dichroism are determined by nonmagnetic optical properties not reflecting the magnetization of the material. Thus, the $180^{\circ}$ 'Kerr rotation' turns out to be a case of polarization rotation in a magnetic material not simply related to magnetic properties. Experimentally, the usual MOKE and the here discussed polarization rotation can be distinguished by an additional analysis of the optical reflectivity.

It would be of interest to determine also the nonlinear Kerr rotation in CeSb. Generally, the nonlinear Kerr effect involves more transitions and will be a more sensitive probe of the electronic structure. Also, the nonlinear Kerr rotation would illustrate the different nature of giant Kerr rotations in linear and nonlinear optics. The theoretical analysis for this follows from previous studies 11,4].

Finally, it is of interest to point out the important formal aspect of our analysis, namely that Eqs. (4) and (6) for the Kerr rotation offer quite generally the possibility of large Kerr rotations. The conditions for this are discussed. Thereby, we analyzed the reflected electrical field with arbitrary ellipticity for the up to now not discussed case of $\operatorname{Re}(\kappa)=0$. We showed the importance of the intraband term in $\chi_{x x}$ and analyzed quantitatively its influence to the $\pm 90^{\circ}$ rotation angle.

We thank J. Schoenes and P. Wachter for stimulating discussions.

1 U. Pustogowa, W. Hübner, and K. H. Bennemann, Phys. Rev. B 49, 10031 (1994).

2 H. A. Wierenga, W. de Jong, M. W. J. Prins, Th. Rasing, R. Vollmer, A. Kirilyuk, H. Schwalbe, and J. Kirschner, Phys. Rev. Lett. 74, 1462 (1995).

3 B. Koopmans, M. Groot Koerkamp, Th. Rasing, and H. van den Berg, Phys. Rev. Lett 74, 3692 (1995).

4 W. Hübner and K. H. Bennemann, Phys. Rev. B. 52, 13411 (1995).

5 W. Reim, J. Schoenes, F. Hulliger, and O. Vogt, J. Magn. Magn. Mater. 54-57, 1401 (1986).

6 R. Pittini, J. Schoenes, O. Vogt, and P. Wachter, Phys. Rev. Lett. 77, 944 (1996).

7 A. N. Yaresko, P. M. Oppeneer, A. Ya. Perlov, V. N. Antonov, T. Kraft, and H. Eschrig, Europhys. Lett. 36, 551 (1996).

8 A. I. Liechtenstein, V. P. Antropov, and B. N. Harmon, Phys. Rev. B 49, 10770 (1994).

9 All electrodynamical relations are given in SI units.

10 M. Groot Koerkamp, Diploma thesis, University of Nijmegen (1995).

11 An equivalent solution of Eq. (1) is given by Groot Ko- 
erkamp 10] with

$$
\varphi=\frac{1}{2} \arctan \left(\frac{2 R e(\kappa)}{1-|\kappa|^{2}}\right)
$$

The solution of Eq. (1) for $\varphi$ and $\varepsilon$ starts with rewriting this complex equation into two real equations for $\operatorname{Re}(\kappa)$ and $\operatorname{Im}(\kappa)$. Whereas Eq. (2) was found by straightforward substitution of $\tan \varepsilon$, another solution is found adding $|\operatorname{Re}(\kappa)|^{2}$ and $|\operatorname{Im}(\kappa)|^{2}$ and subsequently using the addition theorem between $\tan \varphi$ and $\tan 2 \varphi$. Then, the following inversion of $\tan 2 \varphi$ yields an unphysical halving of the definition range to $[-\pi / 4, \pi / 4]$, which is compensated by the angle $\varphi_{0}$ extending the definition range to $[-\pi / 2, \pi / 2]$.

12 Note, these conditions differ from the discussion of Eq. (4) in [3]. Koopmans et al. discussed a $\pm 90^{\circ}$ Kerr rotation for the case of $s$-polarized input light for normal incidence and a magnetization perpendicular to the photon polarization. Note, we define the Kerr rotation as the relative change of polarization directions of reflected to incident photons. Thus, $\kappa=E_{p}(\omega) / E_{s}(\omega)$ and this yields the expected result of $\varphi=0$ for the transversal Kerr geometry (polarization perpendicular to the in-plane magnetization).

13 Note, in magneto-optics the observation of $\Delta l= \pm 1$ for dipole transitions is not required due to the addition of spin-orbit interaction. Furthermore, due to hybridization the discussed bands contain beside the $p$ character also some $f$-electron character.

14 Y. S. Kwon, T. Suzuki, and T. Kasuya, J. Magn. Magn. Mater. 116, 73 (1992).

15 Note, these conditions change when transitions for both majority and minority spin bands have to be considered.

16 H. Feil and C. Haas, Phys. Rev. Lett. 58, 65 (1987).

17 Note the differences between $\mathrm{CeSb}$ and CeBi regarding the ellipticity and the off-diagonal conductivity $\sigma_{x y}$, s. Pittini et al.. Note, CeBi exhibits less structure in $\sigma_{1 x y}$ and $\sigma_{2 x y}$ and a different frequency dependence suggesting different role of important electric dipole transitions.

18 R. Pittini, J. Schoenes, and P. Wachter, to be published (1996).

19 U. Pustogowa, W. Hübner, K. H. Bennemann, Surf. Sc. 307-309, 1129 (1994). 